Random versus Systematic Errors in Reaction Enthalpies Computed Using Semiempirical and Minimal Basis Set Methods

Kromann, Jimmy Charnley; Welford, Alexander; Christensen, Anders S.; Jensen, Jan Halborg

Published in:

ACS Omega

DOI:

10.1021/acsomega.8b00189

Publication date:

2018

Document version

Publisher's PDF, also known as Version of record

Document license:

CC BY

Citation for published version (APA):

Kromann, J. C., Welford, A., Christensen, A. S., \& Jensen, J. H. (2018). Random versus Systematic Errors in

Reaction Enthalpies Computed Using Semiempirical and Minimal Basis Set Methods. ACS Omega, 3(4), $4372-$

4377. https://doi.org/10.1021/acsomega.8b00189 


\title{
Random versus Systematic Errors in Reaction Enthalpies Computed Using Semiempirical and Minimal Basis Set Methods
}

\author{
Jimmy C. Kromann, ${ }^{\dagger}$ Alexander Welford, ${ }^{\dagger}$ Anders S. Christensen, ${ }^{\ddagger}$ and Jan H. Jensen ${ }^{*}{ }^{\dagger} \odot$ \\ ${ }^{\dagger}$ Department of Chemistry, University of Copenhagen, Universitetsparken 5, 2100 Copenhagen, Denmark \\ ${ }^{\ddagger}$ Department of Chemistry, University of Basel, Klingelbergstrasse 80, CH-4056 Basel, Switzerland
}

\section{Supporting Information}

ABSTRACT: The connectivity-based hierarchy ( $\mathrm{CBH}$ ) protocol for computing accurate reaction enthalpies developed by Sengupta and Raghavachari is tested for fast ab initio methods (PBEh-3c, HF-3c, and HF/STO-3G), tight-binding density functional theory (DFT) methods (GFN-xTB, DFTB, and DFTB-D3), and neglect-of-diatomic-differential-overlap (NDDO)-based semiempirical methods (AM1, PM3, PM6, PM6-DH+, PM6-D2, PM6-D3H+, PM6-D3H4X, PM7, and OM2) using the same set of 25 reactions as in the original study. For the $\mathrm{CBH}-2$ scheme, which reflects the change in the immediate chemical environment of all of the heavy atoms, the

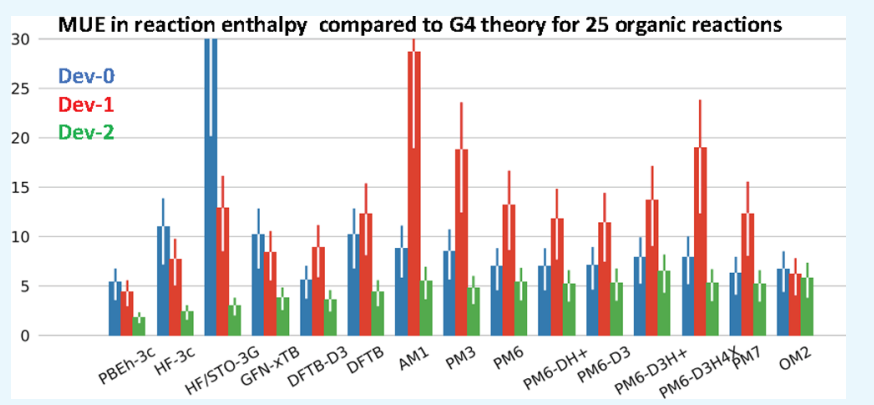
respective mean unsigned error relative to G4 for PBEh-3c, HF-3c, HF/STO-3G, GFN-xTB, DFTB-D3, DFTB, PM3, AM1, PM6, PM6-DH+, PM6-D3, PM6-D3H+, PM6-D3H4X, PM7, and OM2 are 1.9, 2.4, 3.0, 3.9, 3.7, 4.5, 4.8, 5.5, 5.4, 5.3, 5,4, 6.5, 5.3, 5.2, and $5.9 \mathrm{kcal} / \mathrm{mol}$, with a single outlier removed for HF-3c, PM6, PM6-DH+, PM6-D3, PM6-D3H4X, and PM7. The increase in accuracy for the NDDO-based methods is relatively modest due to the random errors in predicted heats for formation.

\section{INTRODUCTION}

Computing accurate reaction enthalpies for large molecules represents a significant challenge to computational chemistry. Sengupta and Raghavachari ${ }^{1}$ recently demonstrated that their connectivity-based hierarchy $(\mathrm{CBH})$ protocol $^{2}$ can be used to compute reaction enthalpies that, on average, are within 1-2 $\mathrm{kcal} / \mathrm{mol}$ of G4 theory, ${ }^{3}$ using density functional theory and triple- $\zeta$ basis sets (DFT/TZV). Although encouraging, the computational cost of the DFT/TZV calculations is still too high to be routinely applied to large biomolecular systems. Here, we test the accuracy of the $\mathrm{CBH}$ approach using computationally more efficient methods, such as DFT/DZV, minimal basis set Hartree-Fock, tight-binding DFT, and neglect-of-diatomic-differential-overlap (NDDO)-based semiempirical methods, using the Sengupta and Raghavachari ${ }^{1}$ data set. As part of the work, we have completely automated the $\mathrm{CBH}$ approach so that it only requires a SMILES string representation of the molecule, which is easily generated using chemical drawing programs, such as ChemDraw.

The paper is organized as follows. We first present the computational methodology including a brief description of the $\mathrm{CBH}$ approach. Next, we discuss the accuracy of the predicted reaction enthalpies as compared to G4 reference values for the 25 reactions used by Sengupta and Raghavachari ${ }^{1}$ and perform an error analysis of outliers by comparing heats of formation (HOF) of the $\mathrm{CBH}$ fragments to G4 values. Finally, we summarize our conclusions and discuss their potential implications for parameterization of semiempirical methods.

\section{RESULTS AND DISCUSSION}

Table 1 shows the mean unsigned error (MUE) relative to G4reaction enthalpies for the parent reaction (Dev-0) and the $\mathrm{CBH}-1$ (Dev-1) and the CBH-2 (Dev-2) correction schemes. In addition, the maximum absolute error for the $\mathrm{CBH}-2$ scheme is also listed. As observed in the Sengupta and Raghavachari ${ }^{1}$ study, $\mathrm{CBH}-1$ correction scheme provides only a modest improvement in accuracy and for many of the semiempirical methods, the error actually increases. We will return the source of the error increase in the next subsection, but for now we will focus on the accuracy of the $\mathrm{CBH}-2$ results.

The MUE of PBEh-3c, which is a dispersion-corrected hybrid DFT/double- $\zeta$ valence method, is $1.9 \mathrm{kcal} / \mathrm{mol}$, which is very similar to the $1.3-2.1 \mathrm{kcal} / \mathrm{mol}$ MUE values obtained by Sengupta and Raghavachari ${ }^{1}$ for dispersion-corrected DFT functionals using the much larger $6-311++\mathrm{G}(3 \mathrm{df}, 2 \mathrm{p})$ basis set and B3LYP/6-311+G(d,p) geometries and frequencies. This is consistent with Sengupta and Raghavachari ${ }^{1}$ 's observation that the Dev-2 error is fairly insensitive to basis set size. Similarly, the MUE of HF-3c, which is a dispersion-corrected HF/ minimal basis set method, is $2.4 \mathrm{kcal} / \mathrm{mol}$, which is only 0.3 $\mathrm{kcal} / \mathrm{mol}$ higher than the value obtained by Sengupta and Raghavachari ${ }^{1}$ using HF/6-311++G(3df,2p)//B3LYP/6$311+\mathrm{G}(\mathrm{d}, \mathrm{p})$. The MUE is computed without reaction 19 ,

Received: January 31, 2018

Accepted: April 12, 2018

Published: April 20, 2018 
Table 1. Dev- $n$ MUE (Mean Unsigned Error) and Max Deviation for Dev-2 for the 25 Reaction Enthalpies in $\mathrm{kcal} / \mathrm{mol}^{a}$

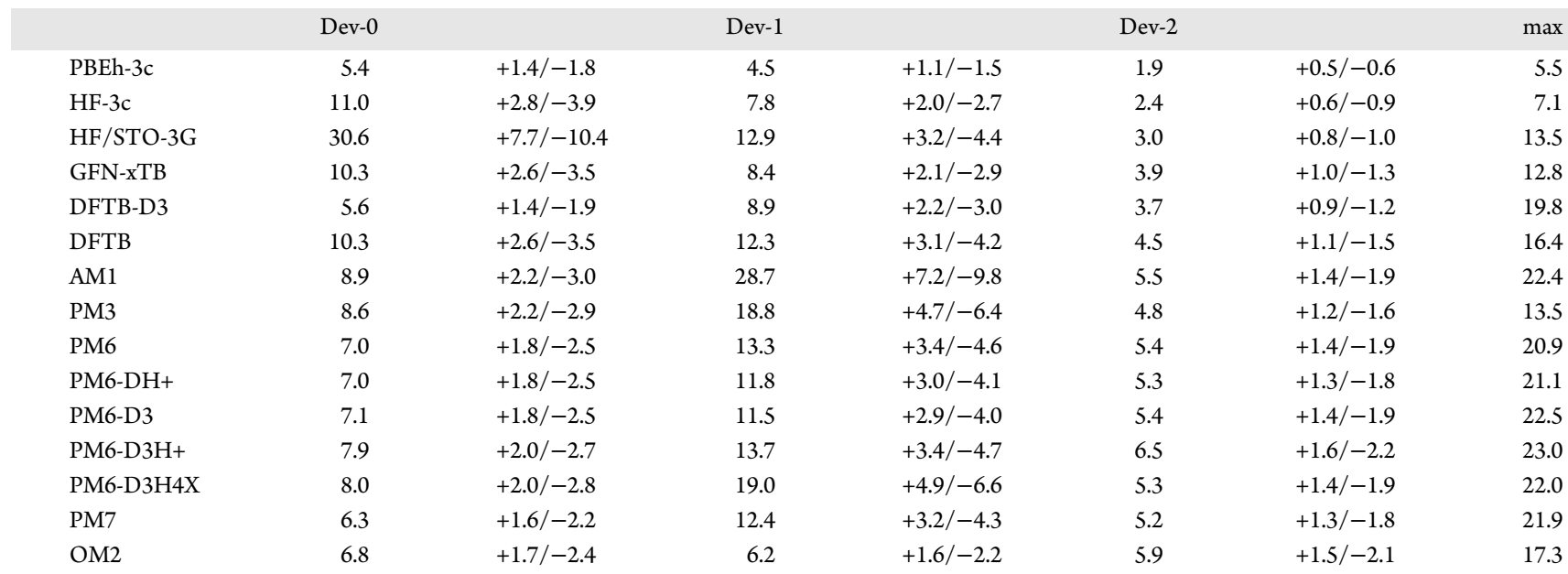

$a_{+} /-$signifies the uncertainty associated with MUE. ${ }^{4}$ Reaction 19 is excluded for HF-3c, and reaction 23 is excluded for PM6 and PM7. The last column lists the maximum absolute error for Dev-2.

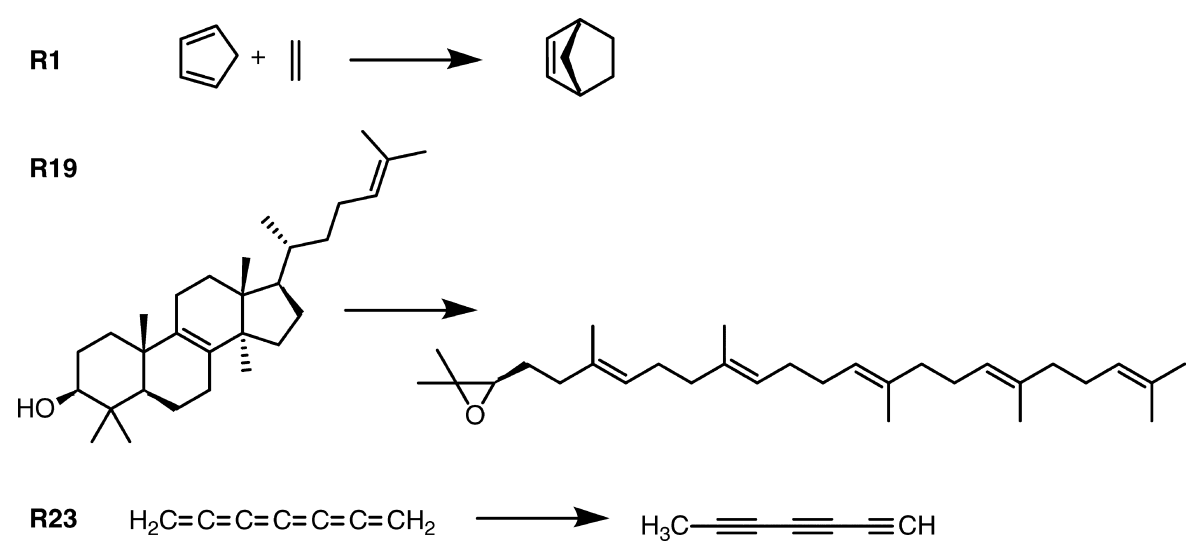

Figure 1. Three of the 25 reactions considered in detail in this paper. The remaining reactions are shown in the Supporting Information (SI).

which is a clear outlier and will be discussed in detail in the next subsection. For comparison, the STO-3G MUE is $3.0 \mathrm{kcal} / \mathrm{mol}$, so empirical corrections (most likely dispersion) contribute to accuracy of the HF-3c method.

GFN-xTB and DFTB-D3 are the most accurate semiempirical methods with MUEs of 3.9 and $3.7 \mathrm{kcal} / \mathrm{mol}$, respectively, followed by DFTB $(4.5 \mathrm{kcal} / \mathrm{mol})$ and PM3 (4.8 $\mathrm{kcal} / \mathrm{mol}$ ), with both pairs of methods being statistically identical (Table S1). The good performance of GFN-xTB and DFTB-D3 is especially remarkable because they are not specifically parameterized against any reaction energies. Comparison of the MUEs for DFTB-D3 and DFTB shows that dispersion corrections lead to noticeably more accurate reaction enthalpies for DFTB, consistent with previous findings. ${ }^{5}$ The remaining NDDO-based methods have statistically identical accuracy, with MUEs in the range of 5.2-5.9 $\mathrm{kcal} / \mathrm{mol}$, with the exception of PM6-D3H+, which has an MUE of $6.5 \mathrm{kcal} / \mathrm{mol}$. The MUEs for PM6, PM6-DH+, PM6D3, PM6-D3H4X, and PM7 are computed without reaction 23, which is a clear outlier and will be discussed in detail in the next subsection. Furthermore, reactions 16 and 22 are omitted for $\mathrm{OM} 2$ because it is not parameterized for fluorine. We note that PM6-D3H+ has an Dev-2 error of $15.0 \mathrm{kcal} / \mathrm{mol}$ and is therefore not considered an outlier. Although the MUE for PM6-D3H+ is higher than that for the other PM6-based methods, it does not lead to any outliers using the Dev-2 method. For the semiempirical methods, there is little correlation between the MUE for parent reaction and the CBH-2-corrected values. Similarly, with an MUE of $6.8 \mathrm{kcal} /$ mol, OM2 performs reasonably well for the parent reactions but worst with $\mathrm{CBH}-2$.

Error Analysis. We start by considering the PM6 result for the first reaction (R1), where the PM6 error is $-0.3 \mathrm{kcal} / \mathrm{mol}$ for the parent reaction and the $\mathrm{CBH}-1$ correction is -12.7 $\mathrm{kcal} / \mathrm{mol}$. PM6 error is quite low for the parent reaction so, ideally, the $\mathrm{CBH}-1$ correction should be close to zero but is in fact quite large in magnitude. To understand the source of this error, we reformulate the $(\Delta \mathrm{CBH}-n(\mathrm{G} 4)-\Delta \mathrm{CBH}-n(\mathrm{LL}))$ correction in terms of errors in heats of formation (HOF, $\left.\Delta H_{\mathrm{f}}^{\circ}\right)$.

$\mathrm{R} 1$ is a Diels-Alder reaction, where two double bonds are converted into four single bonds (Figure 1). The CBH-1 reaction is thus

$$
4 \mathrm{CH}_{4}+2 \mathrm{CH}_{2}=\mathrm{CH}_{2} \rightarrow 4 \mathrm{CH}_{3} \mathrm{CH}_{3}
$$

and

$$
\begin{aligned}
\Delta \mathrm{CBH}-\mathrm{I}_{\mathrm{X}}= & 4 \mathrm{H}_{\mathrm{X}}^{\circ}\left(\mathrm{CH}_{3} \mathrm{CH}_{3}\right)-2 \mathrm{H}_{\mathrm{X}}^{\circ}\left(\mathrm{CH}_{2}=\mathrm{CH}_{2}\right) \\
& -4 \mathrm{H}_{\mathrm{X}}^{\circ}\left(\mathrm{CH}_{4}\right)
\end{aligned}
$$

Therefore the CHB- 1 correction is 


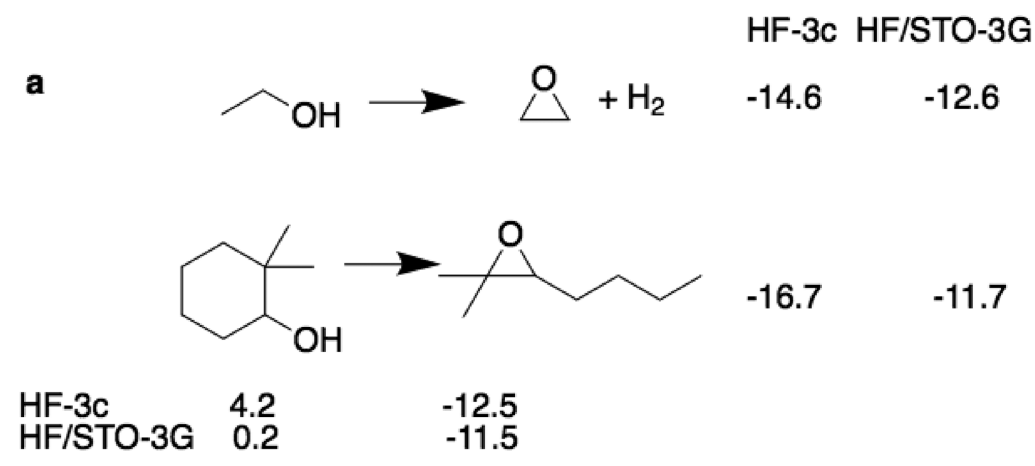

\section{b Product half-reaction}

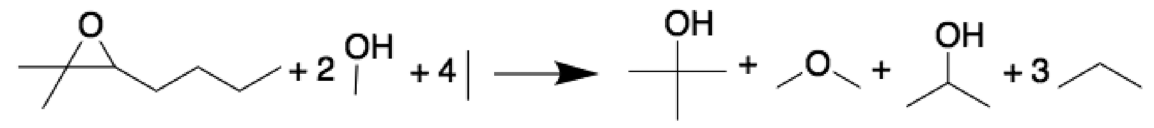

Reactant half-reaction
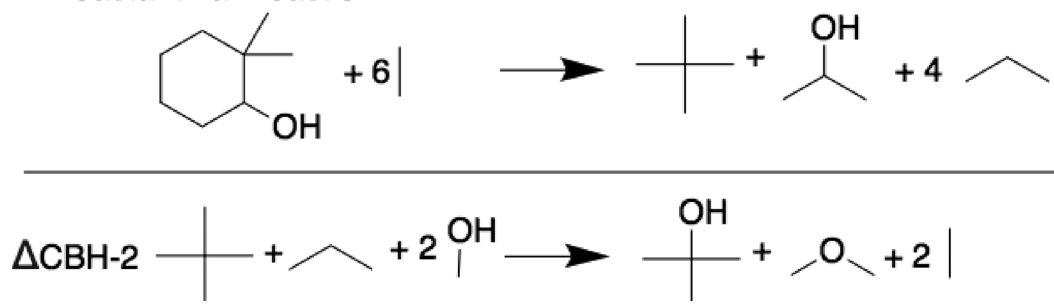

Figure 2. (a) Two model reactions related to reaction 19. Dev-2 values (in $\mathrm{kcal} / \mathrm{mol}$ ) are given on the right hand side, and Dev-2 values for the two half-reactions (shown in (b)), giving rise to the $\triangle \mathrm{CBH}-2$ reaction used to compute Dev-2, are at the bottom.

$$
\begin{aligned}
\Delta \mathrm{CBH}_{-} \mathrm{G}_{\mathrm{G} 4}-\Delta \mathrm{CBH}-\mathrm{I}_{\mathrm{X}} \\
=4 \Delta H^{\circ}\left(\mathrm{CH}_{3} \mathrm{CH}_{3}\right)-2 \Delta H^{\circ}\left(\mathrm{CH}_{2}=\mathrm{CH}_{2}\right)- \\
\quad 4 \Delta H^{\circ}\left(\mathrm{CH}_{4}\right)
\end{aligned}
$$

where, for example

$$
\begin{aligned}
& \Delta H^{\circ}\left(\mathrm{CH}_{3} \mathrm{CH}_{3}\right)=H_{\mathrm{G} 4}^{\circ}\left(\mathrm{CH}_{3} \mathrm{CH}_{3}\right)-H_{\mathrm{LL}}^{\circ}\left(\mathrm{CH}_{3} \mathrm{CH}_{3}\right) \\
& =\Delta H_{\mathrm{f}, \mathrm{G} 4}^{\circ}\left(\mathrm{CH}_{3} \mathrm{CH}_{3}\right)-\Delta H_{\mathrm{f}, \mathrm{LL}}^{\circ}\left(\mathrm{CH}_{3} \mathrm{CH}_{3}\right) \\
& =\Delta \Delta H_{\mathrm{f}}^{\circ}\left(\mathrm{CH}_{3} \mathrm{CH}_{3}\right)
\end{aligned}
$$

The errors in the HOFs for PM6 (and all other methods) relative to G4 are listed in Table $\mathrm{S} 4$ and show that the error in the HOFs relative to $\mathrm{G} 4$ are $\mathrm{CH}_{4}:-5.6, \mathrm{CH}_{2}=\mathrm{CH}_{2}:-3.3$, and $\mathrm{CH}_{3} \mathrm{CH}_{3}:-4.1 \mathrm{kcal} / \mathrm{mol}$. So, the $\mathrm{CBH}-1$ correction is $4(-4.1)$ $-2(-3.3)-4(-5.6)=12.6 \mathrm{kcal} / \mathrm{mol}$ and the $\mathrm{CBH}-1$ correction is large in magnitude because the HOF error for $\mathrm{CH}_{4}$ is somewhat larger than that for $\mathrm{CH}_{3} \mathrm{CH}_{3}$ and, especially, $\mathrm{CH}_{2}=\mathrm{CH}_{2}$. These errors also show that the low error for the parent reaction is clearly fortuitous. If the errors in the HOFs of the reactants and products are similar to those observed for $\mathrm{CH}_{4}, \mathrm{CH}_{2}=\mathrm{CH}_{2}$, and $\mathrm{CH}_{3} \mathrm{CH}_{3}$ then one would expect an error in the reaction enthalpy of about $3-6 \mathrm{kcal} / \mathrm{mol}$ because you go from 2 to 1 molecule.

In contrast, the PBEh-3c error for the parent reaction is 9.0 $\mathrm{kcal} / \mathrm{mol}$, which decreases to $-4.5 \mathrm{kcal} / \mathrm{mol}$ with the $\mathrm{CBH}-1$ correction. The error in the HOFs relative to $\mathrm{G} 4$ are $\mathrm{CH}_{4}: 8.5$, $\mathrm{CH}_{2}=\mathrm{CH}_{2}: 6.0$, and $\mathrm{CH}_{3} \mathrm{CH}_{3}: 14.9 \mathrm{kcal} / \mathrm{mol}$. So the $\mathrm{CBH}-1$ correction is $4(14.9)-2(6.0)-4(8.5)=13.6 \mathrm{kcal} / \mathrm{mol}$, which when subtracted from 9.9 lowers the error in reaction energy to $-4.5 \mathrm{kcal} / \mathrm{mol}$. Clearly, the errors in HOFs are much larger than those for PM6, yet most of the error cancels. The reason is that the magnitude of the HOF error tends to scale with the number and types of bonds in the molecule for ab initio methods. For example, the HOF error of $\mathrm{CH}_{2}=\mathrm{CHCH}_{3}$ is $13.7 \mathrm{kcal} / \mathrm{mol}$, which is approximately the sum of the HOF errors of $\mathrm{CH}_{3} \mathrm{CH}_{3}$ and $\mathrm{CH}_{2}=\mathrm{CH}_{2}$, minus the error for $\mathrm{CH}_{4}$ : $14.9+6.0-8.5=12.4 \mathrm{kcal} / \mathrm{mol}$. This type of error scaling makes $\mathrm{ab}$ initio results very amenable to improvement using the $\mathrm{CBH}$ approach.

We now perform similar analysis of the outliers we removed when computing the Dev-2 MUEs in Table 1, namely, R19 for HF-3c and R23 for PM6, PM6-DH+, and PM7.

For R23, the PM6, PM6-DH+, and PM7 errors for the parent reaction are $0.6,0.6$, and $-1.6 \mathrm{kcal} / \mathrm{mol}$, respectively, whereas the Dev-2 errors are 44.8, 45.0, and $45.7 \mathrm{kcal} / \mathrm{mol}$, much larger in magnitude than other Dev-2 results for these methods. The reaction corresponding to the $\mathrm{CBH}-2$ correction

$$
\begin{gathered}
\mathrm{CH}_{2}=\mathrm{C}=\mathrm{CH}_{2}+2 \mathrm{HC} \equiv \mathrm{CH}+2 \mathrm{CH}_{3} \mathrm{CH}_{3} \rightarrow \\
5 \mathrm{HC} \equiv \mathrm{CCH}_{3}+4 \mathrm{CH}_{2}=\mathrm{CH}_{2}
\end{gathered}
$$

and the corresponding errors in HOFs (Table S4) show that the magnitude of the $\mathrm{CBH}-2$ correction derives from the $\mathrm{HOF}$ error of $\mathrm{CH}_{2}=\mathrm{C}=\mathrm{CH}_{2}(7.7 \mathrm{kcal} / \mathrm{mol})$, which is considerably larger than, and opposite in sign from, the errors in HOF of the remaining fragments $(-1.1$ to $-4.1 \mathrm{kcal} / \mathrm{mol})$.

For R19, the HF-3c Dev-2 error is $-33.5 \mathrm{kcal} / \mathrm{mol}$, much larger in magnitude than other Dev-2 error for HF-3c. For comparison, the corresponding value for $\mathrm{HF} / \mathrm{STO}-3 \mathrm{G}$ is -12.0 
$\mathrm{kcal} / \mathrm{mol}$. In R19, an oxirane ring is formed, which may pose a special challenge to minimal basis sets that may not offer sufficient flexibility to describe the ring strain. Indeed, the Dev2 errors for the first reaction shown in Figure $2 \mathrm{a}$ are -14.6 and $-12.6 \mathrm{kcal} / \mathrm{mol}$ for $\mathrm{HF}-3 \mathrm{c}$ and $\mathrm{HF} / \mathrm{STO}-3 \mathrm{G}$, respectively. Although these errors are similar to the HF/STO-3G Dev-2 error for R19, they are still significantly smaller than the HF-3c Dev-2 error for R19, so we performed similar calculations for the second reaction shown in Figure 2a, which is more similar to R19. The HF-3c and HF/STO-3G errors are -16.4 and $-11.7 \mathrm{kcal} / \mathrm{mol}$, respectively. The source of this increased difference in error between HF-3c and HF/STO-3G can be understood by decomposing the Dev-2 error into contributions from the two "half-reactions" (Figure 2c). The HF-3c and HF/ STO-3G Dev-2 errors for the "product reaction" are very similar $(-12.5$ and $-11.5 \mathrm{kcal} / \mathrm{mol})$, whereas the HF-3c error is $4.0 \mathrm{kcal} / \mathrm{mol}$ higher than that for HF/STO-3G for the reactant reaction. Thus, the higher Dev-2 error observed HF-3c is primarily due to a higher error for the reactant. So, one possible explanation for $-33.5 \mathrm{kcal} / \mathrm{mol}$ error for $\mathrm{HF}-3 \mathrm{c}$ is that roughly $13 \mathrm{kcal} / \mathrm{mol}$ comes from the oxirane ring in the product, whereas the remaining error comes from the four rings in the reactant $(4(4.0)=16 \mathrm{kcal} / \mathrm{mol})$.

HF-3c differs from HF/STO-3G in several ways: HF-3c uses the MINIX basis set instead of STO-3G and has three empirical corrections accounting for dispersion $\left(E_{\text {disp }}\right)$, basis set superposition error $\left(E_{\mathrm{BSSE}}\right)$, and bond length errors $\left(E_{\mathrm{SRB}}\right)$. These three corrections contribute 1.1 and $4.8 \mathrm{kcal} / \mathrm{mol}$ to the errors in the product and reactant half-reactions, respectively. Thus, the larger error compared to HF/STO-3G for the product halfreaction is primarily due to the difference in basis set. This observation is also consistent with the fact that the HF/MINIX Dev-2 error for the R19 is $-24.8 \mathrm{kcal} / \mathrm{mol}$.

\section{CONCLUSIONS AND OUTLOOK}

The connectivity-based hierarchy $(\mathrm{CBH})$ protocol for computing accurate reaction enthalpies developed by Sengupta and Raghavachari ${ }^{1}$ is tested for fast ab initio methods (PBEh-3c, HF-3c, and HF/STO-3G), tight-binding DFT methods (GFNxTB and DFTB), and NDDO-based semiempirical methods (AM1, PM6, PM6-DH+, PM7, and OM2) using the same set of 25 reactions as in the original study. As observed by Sengupta and Raghavachari, ${ }^{1}$ the $\mathrm{CBH}-1$ correction scheme, which reflects the change in bonding, provides only a modest improvement in accuracy and for many of the semiempirical methods the error actually increases. For the $\mathrm{CBH}-2$ scheme, which reflects the change in the immediate chemical environment of all of the heavy atoms, the MUE relative to G4 of $\mathrm{PBEh}-3 \mathrm{c}$ is $1.9 \mathrm{kcal} / \mathrm{mol}$, which is very similar to the $1.3-2.1$ $\mathrm{kcal} / \mathrm{mol}$ MUE values obtained by Sengupta and Raghavachari ${ }^{1}$ using various DFT functionals and triple- $\zeta$ basis sets. The MUE for $\mathrm{HF}-3 \mathrm{c}$, which is a dispersion-corrected $\mathrm{HF} / \mathrm{minimal}$ basis set method, is 2.4 and $3.0 \mathrm{kcal} / \mathrm{mol}$ for HF/STO-3G. The MUE for HF-3c is computed without reaction 19, a clear outlier primarily due to a relatively large error for rings for the HF/ MINIX basis set. GFN-xTB is the most accurate semiempirical method with an MUE of $3.9 \mathrm{kcal} / \mathrm{mol}$, followed by DFTB (4.5 $\mathrm{kcal} / \mathrm{mol})$ and PM3 $(4.8 \mathrm{kcal} / \mathrm{mol})$. The remaining NDDObased methods, AM1, PM6, PM6-DH+, PM7, and OM2, have statistically equal accuracy with MUEs in the range of 5.2-5.9 $\mathrm{kcal} / \mathrm{mol}$. The MUEs for PM6, PM6-DH+, and PM7 are computed without reaction 23 , which is a clear outlier due to an unusually large error in the heat of formation (HOF) compared to G4 for one of the components in the correcting reaction.

Although the accuracy is lower for the minimal basis set and semiempirical methods are lower than for PBEh-3c, they are significantly more computationally efficient with $\mathrm{HF}-3 \mathrm{c}$ and HF/STO-3G being roughly 10-50 times faster and the semiempirical methods being roughly 1000 times faster, depending on system size. It is worth noting that GFN-xTB is roughly 10 times faster than the other semiempirical methods, which can lead to significant time savings when dealing with thousands of molecules. However, significant changes in bonding and/or unusual bonding still present challenge to these faster methods, as evidenced by the presence of outliers.

More generally, our analyses show that although the errors in HOF computed by ab initio and tight-binding DFT methods tend to be larger in magnitude than those for the NDDO-based semiempirical methods, the magnitude tends to scale systematically with the number and types of bonds in the molecule, which make them very amenable to improvement using the $\mathrm{CBH}$ approach. The NDDO-based semiempirical methods are optimized by reducing the absolute HOF error independent of system size and, as a result, the HOF error of an individual molecule tends to be relatively random both in sign and magnitude, which makes it less amenable to improvement using the $\mathrm{CBH}$ approach. A better approach may be to parameterize the semiempirical methods by minimizing the $\mathrm{CBH}$-corrected error and presenting the $\mathrm{CBH}$-corrected energies to the user. For example, although NDDO-based methods would parameterize by minimizing the HOF error of propane $(\Delta \mathrm{HOF}-$ (propane)), a $\mathrm{CBH}$-1-based scheme would minimize $[\Delta \mathrm{HOF}$ (propane) $-\Delta \mathrm{HOF}($ ethane $)-\Delta \mathrm{HOF}($ methane $)]$ and present the $\mathrm{CBH}-1$-corrected HOF to the user. This approach might make it easier to find more generally applicable parameters that better and systematically minimize the error because the underlying HF approach "naturally" gives larger errors for larger systems, and we are no longer asking the parameters to "undo that" by searching for small errors independent of system size.

We implemented the $\mathrm{CBH}-1$ and $\mathrm{CBH}-2$ correction schemes in a program called fragreact, which identifies the appropriate fragments for a given parent reaction, generates input files for the fragments (if needed), determines the balanced equation for the correction reaction, and, in general, automates the entire process.

\section{COMPUTATIONAL DETAILS}

The connectivity-based hierarchy $(\mathrm{CBH})$ protocol has been described in detail elsewhere ${ }^{1,2}$ and is only summarized here. The aim of the method is to approximate the reaction enthalpy of a reaction (the "parent reaction") at a high level of theory (here taken to be G4) by computing the reaction enthalpy using a faster, lower level method ("LL" in the equations below) and subtracting a correction.

$$
\mathrm{PR}(\mathrm{G} 4) \approx \mathrm{PR}(\mathrm{LL})+(\Delta \mathrm{CBH}-n(\mathrm{G} 4)-\Delta \mathrm{CBH}-n(\mathrm{LL}))
$$

Here, $\Delta \mathrm{CBH}-n(\mathrm{G} 4)$ is the G4-reaction enthalpy computed for a reaction involving smaller fragments that reflect the change in bonding of the parent reaction where the size of the fragment is defined by $n$ and similarly for $\Delta \mathrm{CBH}-n(\mathrm{LL})$. For $\Delta \mathrm{CBH}-1$, the reaction involves fragments made of two heavy (nonhydrogen) atoms and reflects the change in bonding, whereas for $\Delta \mathrm{CBH}$ - 
2 , reaction involves fragments made from 3 to 5 heavy atoms and reflects the change in the immediate chemical environment of all of the heavy atoms.

Following Sengupta and Raghavachari, ${ }^{1}$ we evaluate the error relative to the parent reaction using G4-reaction energies as reference.

$$
\begin{aligned}
& \text { Dev-0 }=\text { PR }(\mathrm{G} 4)-\operatorname{PR}(\mathrm{LL}) \\
& \text { Dev-1 }=\text { Dev-0 }-(\Delta \mathrm{CBH}-1(\mathrm{G} 4)-\Delta \mathrm{CBH}-1(\mathrm{LL})) \\
& \text { Dev-2 }=\text { Dev-0 }-(\Delta \mathrm{CBH}-2(\mathrm{G} 4)-\Delta \mathrm{CBH}-2(\mathrm{LL}))
\end{aligned}
$$

We use the G4 reference energies for 25 reactions from the study of Sengupta and Raghavachari ${ }^{1}$ (referred to as R1-R25). Note that for R19, R20, and R22, DLPNO-CCSD(T)/ccpVTZ is used instead of G4.

We implemented the $\mathrm{CBH}-1$ and $\mathrm{CBH}-2$ correction schemes in a program called fragreact, ${ }^{6}$ heavily depending on RDKit, ${ }^{7}$ which identifies the appropriate fragments for a given parent reaction, generates input files for the fragments (if needed), determines the balanced equation for the correction reaction, and, in general, automates the entire process.

Although our fragreact results generally agree with those of Sengupta and Raghavachari, ${ }^{1}$ we did obtain slightly different $\Delta \mathrm{CBH}-2$ correction reactions for $\mathrm{R} 18, \mathrm{R} 19$, and $\mathrm{R} 23$, but the effect on the mean unsigned error computed at the B3LYP/6$311+\mathrm{G}(\mathrm{d}, \mathrm{p})$ level of theory is $0.1 \mathrm{kcal} / \mathrm{mol}$, as described in SI. All fragment G4 energies were recomputed as part of this study.

In this study, we test the accuracy of the $\mathrm{CBH}-1$ and $\mathrm{CBH}-2$ schemes for PBEh-3c, ${ }^{8}$ HF-3c, ${ }^{9}$ HF $/$ STO-3G, ${ }^{10}$ GFN-xTB, ${ }^{11}$ DFTB, ${ }^{12}$ DFTB-D3, ${ }^{13}$ AM1, ${ }^{14}$ PM3,${ }^{15}$ PM6, ${ }^{16}$ PM6-DH+, ${ }^{17}$ PM6-D3, ${ }^{18}$ PM6-D3H+, ${ }^{19}$ PM6-D3H4X, ${ }^{20}$ PM7, ${ }^{21}$ and OM2. ${ }^{2,23}$ In the study of Sengupta and Raghavachari, ${ }^{1}$ all results are based on single-point energy calculations based on structures optimized with B3LYP/6-311+G(d,p) and corresponding rigid rotor/harmonic oscillator enthalpy corrections. We used geometries optimized at the respective levels, where the geometry optimizations of the parent reaction molecules are initiated from the B3LYP/6-311+G(d,p) geometries supplied by Sengupta and Raghavachari ${ }^{1}$ (some molecules related to R2, R5, and R16 were missing and build from scratch). Enthalpy corrections are not included for the NDDObased methods because they are parameterized to reproduce enthalpies of formation. We computed enthalpies of formation, for analysis purposes, using the method outlined by Curtiss et al. $^{24}$ The PBEh-3c and HF-3c are performed with ORCA, ${ }^{25}$ the DFTB calculations with GAMESS, ${ }^{26}$ the XTB calculations with the xTB program, ${ }^{11}$ the OM2 calculations with $\mathrm{MNDO},{ }^{27}$ and the remaining NDDO-based calculations are performed with MOPAC. ${ }^{28}$

\section{ASSOCIATED CONTENT}

\section{S Supporting Information}

The Supporting Information is available free of charge on the ACS Publications website at DOI: 10.1021/acsomega.8b00189.

Method MUE on Dev0-2 (Section S1); reactions (Section S2); fragmentation example (Section S3); overview of methods with Dev- $n$ (Section S4); statistically significant test (Section S5); difference in correction schemes (Section S6); heat of formation deviation (Section S7) (PDF)

\section{AUTHOR INFORMATION}

\section{Corresponding Author}

*E-mail: jhjensen@chem.ku.dk.

ORCID

Jan H. Jensen: 0000-0002-1465-1010

\section{Notes}

The authors declare no competing financial interest.

The Cartesian coordinates of the molecules used in this study can be found here: https://doi.org/10.6084/m9.figshare. 5822061.

\section{ACKNOWLEDGMENTS}

J.H.J. thanks Robert Paton (@bobbypaton) and @Frauenchemie for helpful Twitter discussions.

\section{REFERENCES}

(1) Sengupta, A.; Raghavachari, K. Solving the Density Functional Conundrum: Elimination of Systematic Errors to Derive Accurate Reaction Enthalpies of Complex Organic Reactions. Organic Letters [Online], 2017, Vol. 19, no. 10, pp. 2576-2579. https://doi.org/10. 1021\%2Facs.orglett.7b00891.

(2) Ramabhadran, R. O.; Raghavachari, K. Theoretical Thermochemistry for Organic Molecules: Development of the Generalized Connectivity-Based Hierarchy. Journal of Chemical Theory and Computation [Online], 2011, Vol. 7, no. 7, pp. 2094-2103. https:// doi.org/10.1021\%2Fct200279q.

(3) Curtiss, L. A.; Redfern, P. C.; Raghavachari, K. Gaussian-4 Theory. The Journal of Chemical Physics [Online], 2007, Vol. 126, no. 8, p. 084108. https://doi.org/10.1063\%2F1.2436888.

(4) Jensen, J. H. Which Method is More Accurate? or Errors Have Error Bars [Online], 2017. https://doi.org/10.7287/peerj.preprints. 2693v1.

(5) Gruden, M.; Andjeklović, L.; Jissy, A. K.; Stepanović, S.; Zlatar, M.; Cui, Q.; Elstner, M. Benchmarking Density Functional Tight Binding Models for Barrier Heights and Reaction Energetics of Organic Molecules. Journal of Computational Chemistry [Online], 2017, Vol. 38, no. 25, pp.2171-2185. https://doi.org/10.1002/jcc. 24866.

(6) Kromann, J. C.; Welford, A. GitHub: Reaction Fragmentation Scheme Using Connectivity-based Hieracy [Online], 2017. https:// github.com/jensengroup/fragreact.

(7) Landrum, G. Rdkit: Open-Source Cheminformatics [Online]. http://www.rdkit.org.

(8) Grimme, S.; Brandenburg, J. G.; Bannwarth, C.; Hansen, A. Consistent Structures and Interactions by Density Functional Theory with Small Atomic Orbital Basis Sets. The Journal of Chemical Physics [Online], 2015, Vol. 143, no. 5, p. 054107. https://doi.org/10. $1063 \% 2 \mathrm{~F} 1.4927476$.

(9) Sure, R; Grimme, S. Corrected Small Basis Set Hartree-Fock Method for Large Systems. J. Comput. Chem. 2013, 34, 1672-1685.

(10) Hehre, W. J.; Stewart, R. F.; Pople, J. A. Self-Consistent Molecular-Orbital Methods. i. Use of Gaussian Expansions of SlaterType Atomic Orbitals. The Journal of Chemical Physics [Online], 1969, Vol. 51, no. 6, pp. 2657-2664. https://doi.org/10.1063\%2F1. 1672392.

(11) Grimme, S.; Bannwarth, C.; Shushkov, P. A robust and Accurate Tight-Binding Quantum Chemical Method for Structures, Vibrational Frequencies, and Noncovalent Interactions of Large Molecular Systems Parametrized for all spd-Block Elements $(\mathrm{z}=1-86)$. Journal of Chemical Theory and Computation [Online], 2017, Vol. 13, no. 5, pp. 1989-2009. https://doi.org/10.1021\%2Facs.jctc.7b00118.

(12) Gaus, M.; Cui, Q.; Elstner, M. DFTB3: Extension of the SelfConsistent-Charge Density-Functional Tight-Binding Method (SCCDFTB). Journal of Chemical Theory and Computation [Online], 2011, Vol. 7, no. 4, pp. 931-948. https://doi.org/10. $1021 \% 2$ Fct100684s. 
(13) Zhechkov, L.; Heine, T.; Patchkovskii, S.; Seifert, G.; Duarte, H. A. An Efficient a Posteriori Treatment for Dispersion Interaction in Density-Functional-Based Tight Binding. Journal of Chemical Theory and Computation [Online], 2005, Vol. 1, no. 5, pp. 841-847. https:// doi.org/10.1021/ct050065y.

(14) Dewar, M. J. S.; Zoebisch, E. G.; Healy, E. F.; Stewart, J. J. P. Development and use of quantum mechanical molecular models. 76. AM1: a new general purpose quantum mechanical molecular model. J. Am. Chem. Soc. 1985, 107, 3902-3909.

(15) Stewart, J. J. P. Optimization of Parameters for Semi-Empirical Methods I-Method. J. Comput. Chem. 1989, 10, 209-220.

(16) Stewart, J. J. P. Optimization of parameters for semiempirical methods V: Modification of NDDO approximations and application to 70 elements. J. Mol. Model. 2007, 13, 1172-1213.

(17) Korth, M. Third-Generation Hydrogen-Bonding Corrections for Semiempirical QM Methods and Force Fields. J. Chem. Theory Comput. 2010, 6, 3308-3816.

(18) Grimme, S.; Antony, J.; Ehrlich, S.; Krieg, H. A Consistent and Accurate Ab Initio Parametrization of Density Functional Dispersion Correction (DFT-d) for the 94 Elements h-pu. The Journal of Chemical Physics [Online], 2010, Vol. 132, no. 15, p. 154104. https:// doi.org/10.1063/1.3382344.

(19) Kromann, J. C.; Christensen, A. S.; Steinmann, C.; Korth, M.; Jensen, J. H. A Third-Generation Dispersion and Third-Generation Hydrogen Bonding Corrected PM6 Method: PM6-d3h+. PeerJ [Online], 2014, Vol. 2, p. e449. https://doi.org/10.7717/peerj.449.

(20) Brahmkshatriya, P. S.; Dobes, P.; Fanfrlik, J.; Rezac, J.; Paruch, K.; Bronowska, A.; Lepsãk, M.; Hobza, P. Quantum Mechanical Scoring: Structural and Energetic Insights into Cyclin-Dependent Kinase 2 Inhibition by Pyrazolo[1,5-a]Pyrimidines [Online], 2013, Current Computer - Aided Drug Design, Vol. 9, no. 1, pp. 118-129. https://doi.org/10.2174/157340913804998784.

(21) Stewart, J. J. P. Optimization of Parameters for Semiempirical Methods VI: More Modifications to the NDDO Approximations and Re-Optimization of Parameters. Journal of Molecular Modeling [Online], 2012, Vol. 19, no. 1, pp. 1-32. https://doi.org/10. 1007\%2Fs00894-012-1667-x.

(22) Weber, W. Ein Neues Semiempirisches NDDO-Verfahren Mit Orthogonalisierungskorrekturen: Entwicklung Des Modells, Parametrisierung Und Anwendungen. Ph.D. Thesis, 1996.

(23) Weber, W.; Thiel, W. Orthogonalization Corrections for Semiempirical Methods. Theoretical Chemistry Accounts: Theory, Computation, and Modeling (Theoretica Chimica Acta) [Online], 2000, Vol. 103, no. 6, pp. 495-506. https://doi.org/10. $1007 \% 2 \mathrm{Fs} 002149900083$.

(24) Curtiss, L. A.; Raghavachari, K.; Redfern, P. C.; Pople, J. A. Assessment of Gaussian-2 and Density Functional Theories for the Computation of Enthalpies of Formation. The Journal of Chemical Physics [Online], 1997, Vol. 106, no. 3, pp. 1063-1079. https://doi. org/10.1063\%2F1.473182.

(25) Neese, F. ORCA Program System [Online], 2011, Wiley Interdisciplinary Reviews: Computational Molecular Science, Vol. 2, no. 1, pp. 73-78. https://doi.org/10.1002\%2Fwcms.81.

(26) Schmidt, M. W.; Baldridge, K. K.; Boatz, J. A.; Elbert, S. T.; Gordon, M. S.; Jensen, J. H.; Koseki, S.; Matsunaga, N.; Nguyen, K. A.; Su, S.; Windus, T. L.; Dupuis, M.; Montgomery, J. A. General atomic and molecular electronic structure system. J. Comput. Chem. 1993, 14, 1347-1363.

(27) Dewar, M. J. S.; Thiel, W. Ground states of molecules. 38. The MNDO method. Approximations and parameters. J. Am. Chem. Soc. 1977, 15, 4899-4907.

(28) Stewart, J. J. P. MOPAC2016 [Online], 2016, Stewart Computational Chemistry, Colorado Springs, CO. http:// openmopac.net. 